\title{
La ville, corps malade : Besançon 1544
}

\section{Nicolas Sarzeaud}

\section{OpenEdition}

\section{Journals}

Édition électronique

URL : https://journals.openedition.org/tc/9528

DOI : $10.4000 /$ tc. 9528

ISSN : 1952-420X

\section{Éditeur}

Éditions de l'EHESS

\section{Édition imprimée}

Date de publication : 30 octobre 2018

Pagination : 80-97

ISBN : 2-7132-2751-6

ISSN : 0248-6016

\section{Référence électronique}

Nicolas Sarzeaud, «La ville, corps malade : Besançon 1544 », Techniques \& Culture [En ligne], 70 | 2018, mis en ligne le 06 décembre 2020, consulté le 29 septembre 2022. URL : http://

journals.openedition.org/tc/9528; DOI : https://doi.org/10.4000/tc.9528 


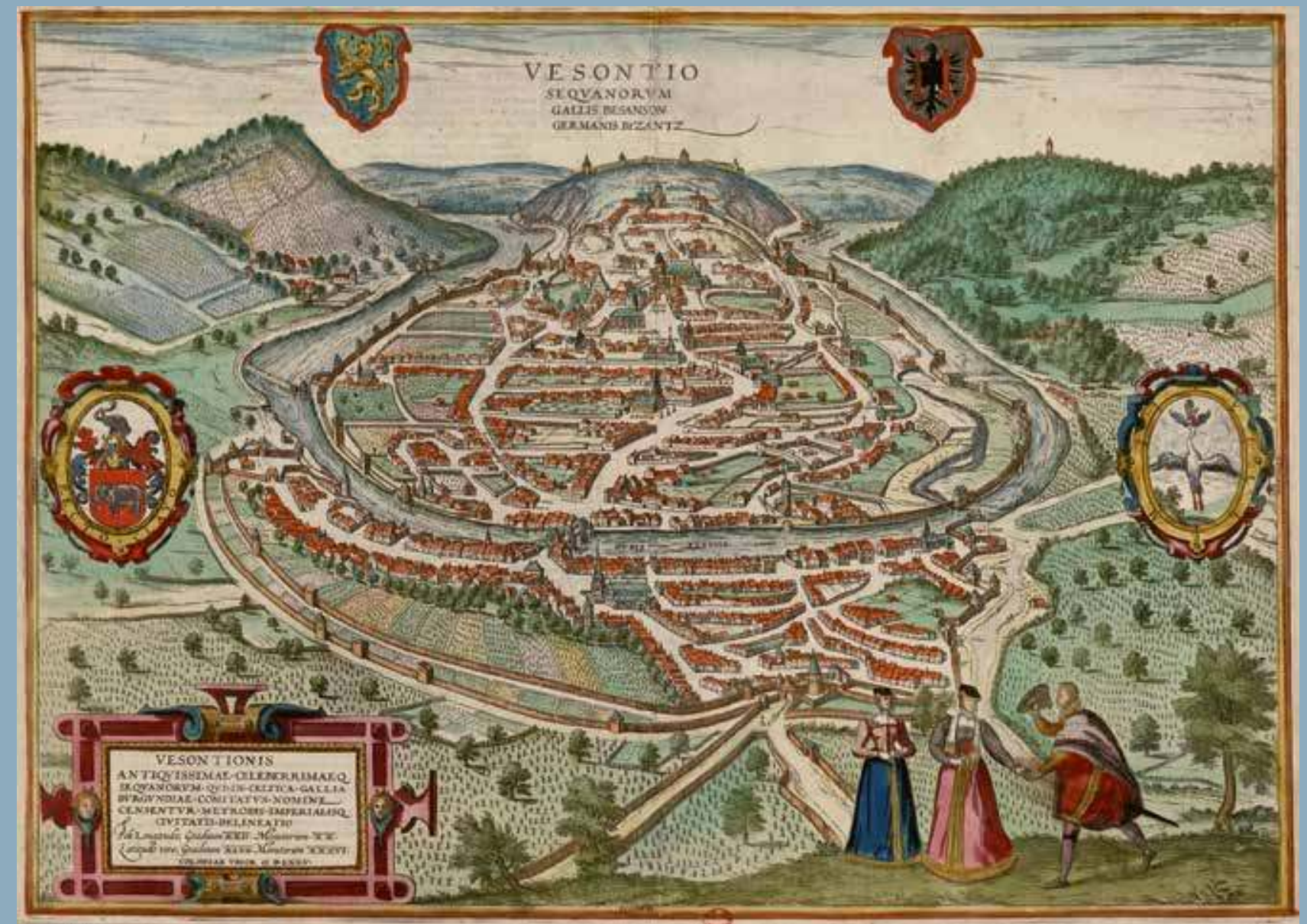




\section{La ville, corps malade : Besançon 1544}

On a un peu de peine à se représenter la peste. On se figure des sons de crécelles, une odeur de charnier, un ciel sombre d'apocalypse, celui décrit par Hermann Hesse dans Narcisse et Goldmund (2010 [1930] : 178-179):

«Toute la région, tout le vaste pays était sous un nuage de mort, sous un voile d'horreur, d'angoisse, qui assombrissait les âmes, et le pire, ce n'étaient pas les maisons désertes, les chiens qui pourrissaient morts de faim à la chaîne, les morts restés sans sépulture, les enfants qui mendiaient, les fosses communes devant les villes. Le pire, c'étaient les vivants qui, sous le fardeau de l'effroi et de l'angoisse mortelle, semblaient avoir perdu leurs yeux et leurs âmes. »

Les écrivains ne furent pas les seuls à faire ces rêves tragiques, les historiens ont cherché dans la documentation et dans l'art de la fin du Moyen Âge les traces du grand traumatisme que dut être la peste. Depuis l'ouvrage de Millard Meiss (2013 [1951]) la question a prospéré: qu'est-ce qu'un «art de crise» (Baschet 1994: 45) ? Est-ce une imagerie funèbre, composée de visions infernales? Est-ce l'expression d'une religiosité exacerbée, tendue vers l'espoir d'un Salut?

Nous sommes en juin 1544 à Besançon, la ville étouffe. Les gouverneurs, collège élu par les citoyens, ont nommé des barbiers et des fossoyeurs pour les pestiférés, prononcé des mesures d'exclusion, annulé des cérémonies publiques. Malgré ce, les morts se comptent vraisemblablement en milliers; lors de l'épidémie précédente en 1533, les gouverneurs dressaient le bilan de cinq années de peste pendant lesquelles étaient «trespassez plus de quatre mil personnes (BB15: fo362r)», plus d'un tiers des habitants d'une ville qui en compte entre huit et douze milles (Delsalle 2004: 159).

Que faire? Alors que l'été arrive et avec lui les chaleurs favorables à la propagation de la maladie, les gouverneurs font réaliser une image votive: ce n'est pas une vision d'apocalypse, ni une bouche d'enfer, ni un Christ-juge, mais une «cité en cyre, la plus triomphante que 
possible seroit », déposée devant le Saint Suaire, dans la cathédrale Saint-Étienne. Il fallait une image pour que la ville se présente et se donne efficacement à la relique. Le matériau, la cire, traditionnellement utilisé par les fidèles souffrant pour se vouer à une figure protectrice, donne à la ville une corporéité. C'est cet acte d'image qui va nous occuper et avec lui l'étonnant effet de l'épidémie; pousser la ville à dresser d'elle-même un portrait efficace ${ }^{1}$.

\section{Vouer le corps de la cité}

Le 16juin 1544, les gouverneurs de Besançon écrivent dans leur registre:

«[P]uis quatre ou cinq ans ença continuellement la contagieuse maladie de peste + a tousjours regné et regne en ceste cite, sans y avoir peuys remedier, non obstans toutes polices, diligences et bonnes provisions. » (BB24: f०55v.)

Mention de la peste dans les délibérations des gouverneurs Archives municipales de Besançon

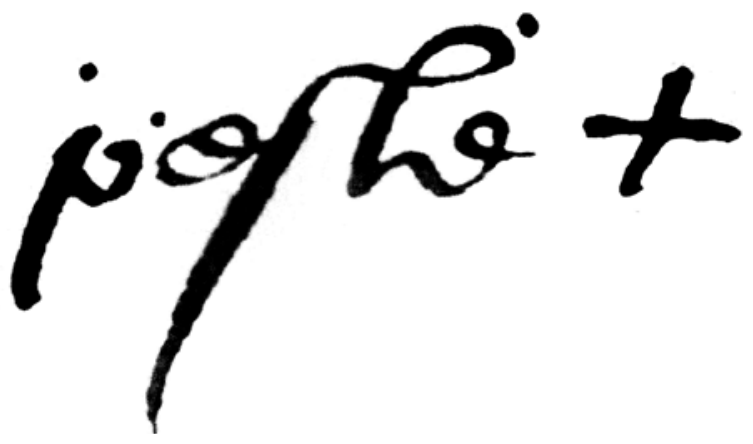

Peste! Il faudrait comme le secrétaire de la Ville marquer le mot d'une petite croix, pour ne pas appeler le mal que l'on énonce. Ce registre compte de nombreuses occurrences de ce terme, mais c'est la seule marquée d'une croix apotropaïque. Voilà un premier indice de la performativité particulière d'un texte dans lequel les gouverneurs engagent la ville derrière eux et devant Dieu:

«[Il n’y a] pour le présent aultre remède ou moyen que de recourir à Dieu le créateur. Et [les gouverneurs] ont advisés de faire dresser et vouher tant en leurs noms que de tout le corps de la cité une confrairie au très précieux et très digne saint Suayre. Afin [que] Dieu le créateur, par son infinie bonté, profunde clémence et exubérante miséricorde, par la vertu et esficace dudit très digne saint Suayre qu'[il] nous a délaissé, en recordation de sa très doloreuse mort et passion, vueille appaiser son ire et préserver ladite cité d'icelle maladie de peste et tous aultres inconvéniens. » (Ibid.) 


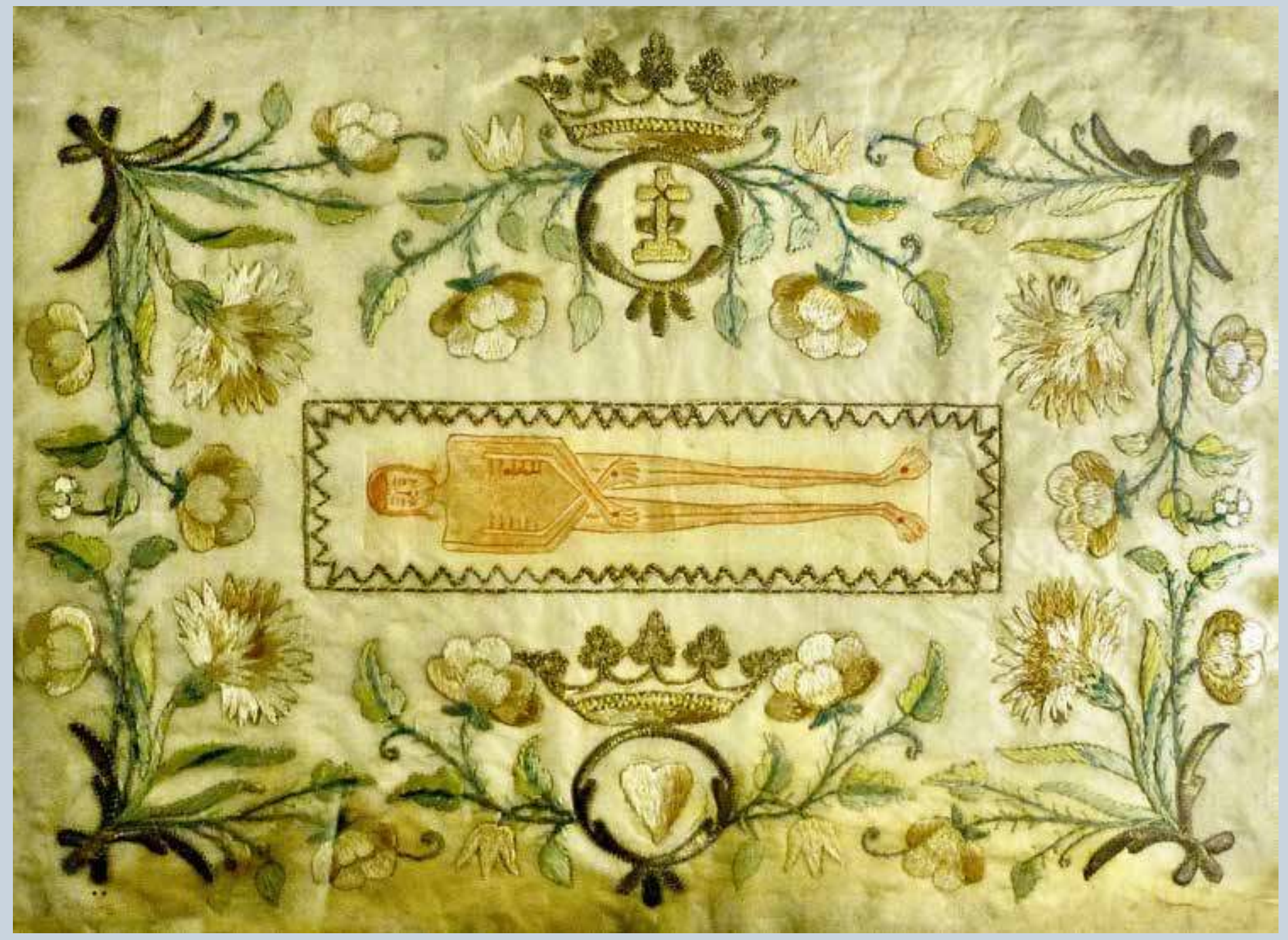


Il s'agit d'un contrat votif; afin d'apaiser la colère de Dieu et d'obtenir sa protection, la cité se voue au Saint Suaire, linge funéraire du Christ portant son empreinte et conservé dans la cathédrale Saint-Étienne depuis 1523 (Nicolotti 2015). Pour obtenir une guérison générale, la cité doit se présenter comme un corps solidaire: les habitants de Besançon se trouvent incorporés au vœu des gouverneurs et à la confrérie du Saint Suaire, institution dont le propre est précisément d'établir une communauté de chair. Ils sont aussi incorporés à une image, dont la réalisation est décidée par les gouverneurs le 21 juin, jour du premier office de la confrérie:

«Messires ont conclu que l'on feroit une cité en cyre, la plus triomphante que possible seroit, laquelle l'on offreroit en la chappelle dudit Saint Suayre. » (Ibidem: for.)

Comme un fidèle qui dépose devant la relique une image en cire de ses organes malades en échange d'une guérison (Didi-Hubermann 2006), la ville dépose une image d'elle-même, triomphante; c'est un ex-voto propitiatoire qui doit préfigurer la fin de la crise. Il est offert au Saint Suaire le 26juillet, à l'occasion d'une messe donnée aux gouverneurs et notables de Besançon en l'église Saint-Étienne (ibidem: fo73v) ${ }^{2}$.

Réalisée dans un matériau peu pérenne, l'offrande a aujourd'hui disparu. Pour autant, il s'agissait d'un objet de valeur: le 10 juillet, Girard d'Argent et Bastien de Roset se partagent 10 francs pour avoir «fait et construict en cyre la cité pour offrir devant le précieux Saint Suaire», Jean et Louis Saulget reçoivent 15 francs et 9 gros «pour la cyre employée et mise en œuvre» (CC102: f119r). La somme est conséquente: plus de 25 francs, soit 309 gros, plusieurs mois d'un salaire ordinaire (Étienne Jobert, à qui échoit la tâche ingrate de fossoyeur des pestiférés, reçoit 4 francs pour le mois d'avril 1544, ibid. : fo178v). La somme allouée aux Saulget, 189 gros, ne spécifie pas le prix de la cire mais laisse imaginer un objet volumineux; dans les mêmes comptes les gouverneurs achètent deux cierges pour 4 gros (ibid. : f^11lv).

Parmi les quatre artisans cités, le mieux connu est Girard d'Argent, peintre travaillant pour le chapitre et pour la Ville 3 . Premier nommé, il a pu concevoir l'image, que deux témoignages ultérieurs décrivent comme un «tableau votif » ${ }^{4}$; il devait s'agir d'un grand panneau de cire gravé ou moulé en bas-relief. Si la documentation ne précise pas comment a été représentée la cité, on peut s'appuyer sur deux vues de Besançon plus tardives: la première a été peinte par Pierre d'Argent le Vieux, fils de Girard (cf. image d'ouverture) circa 1570 et publiée en 1575, la seconde a été commandée par la Ville à Samson Bruley en 1615 pour remplacer l'ex-voto de 1544 dans la chapelle du Saint-Suaire (voir infra). La peinture de 1615 reprenait probablement la mise en page du tableau de cire auquel elle se substituait et les importantes ressemblances avec la peinture de Pierre d'Argent semblent confirmer que ces deux vues de Besançon s'inspiraient d'un même prototype. Les deux œuvres s'ouvrent au bord inférieur sur une même bande de terre et donnent à voir la ville sous un même angle, axées sur le rocher où s'élève la cathédrale Saint-Étienne et couronnées par les blasons de l'Empire et de la Ville. Il faut donc imaginer, en 1544, une vue cavalière de ville gravée

Besançon à vol d'oiseau, Samson Bruley, 1615 , huile sur bois dans la cire, représentant ses principales rues et architectures, inscrites dans la boucle du Doubs et dans le paysage vallonné alentour. Sous un angle similaire, elle devait faire trôner la cathédrale dans laquelle se conservait le Saint Suaire et afficher les emblèmes de la cité. 


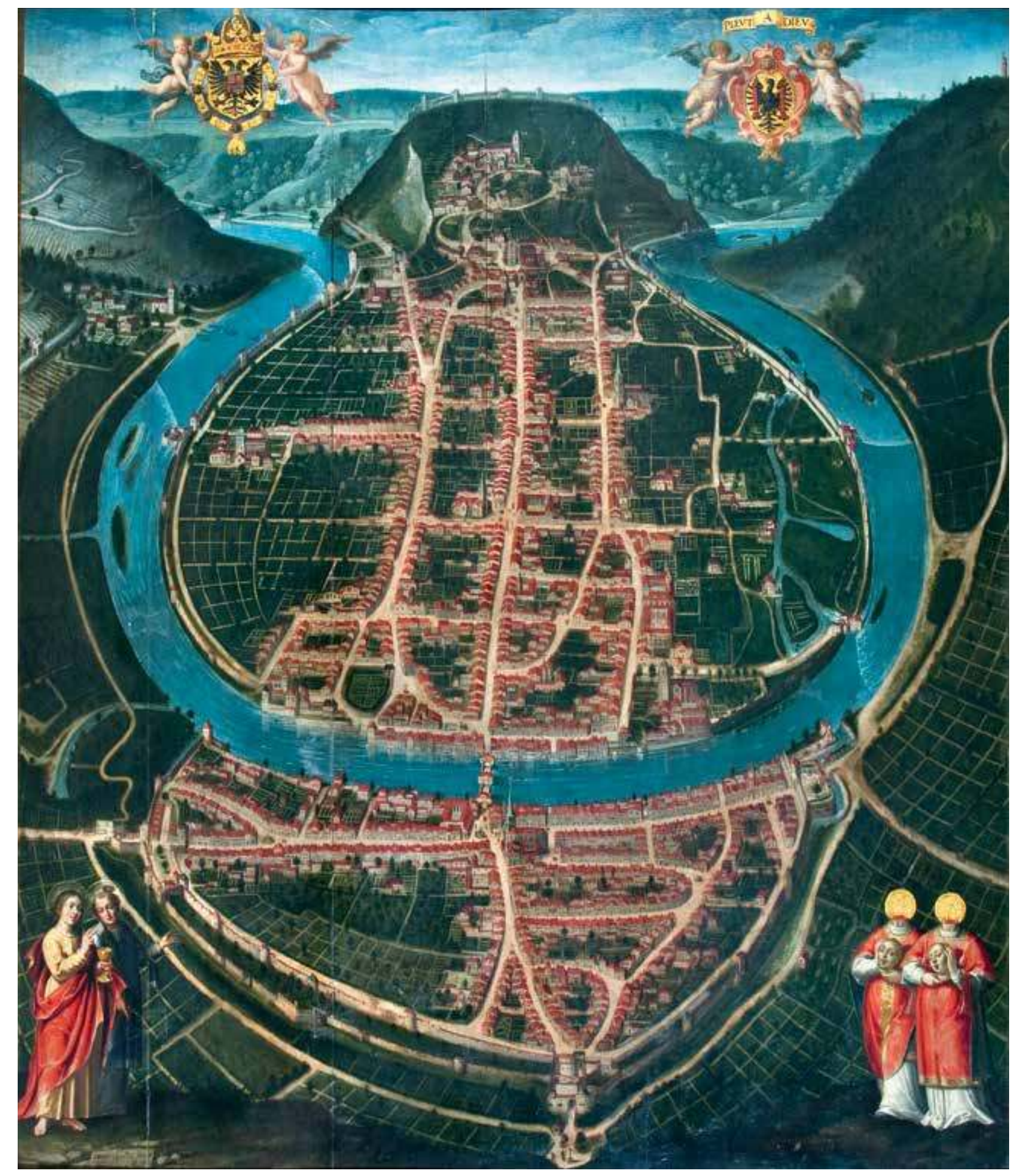




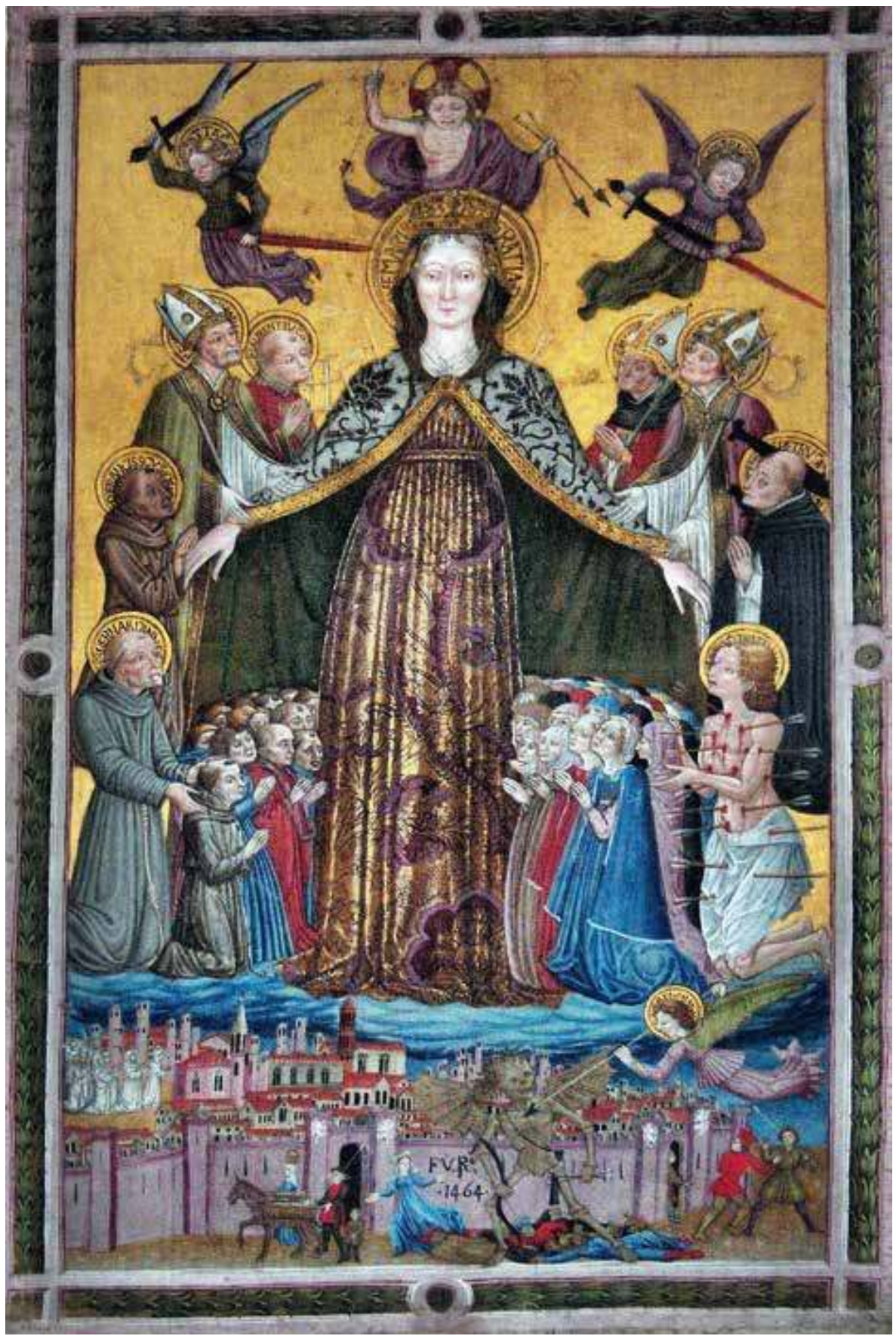

\section{Le corps du lieu}

On s'est efforcé de faire émerger une incidence de l'épidémie sur la couleur générale de l'image médiévale; les œuvres produites en réaction à la peste sont des témoins privilégiés de «l'art de crise». On les retrouve à partir des premières récurrences de la Grande Peste de 1347 et elles représentent essentiellement les saints Roch, Sébastien et des Vierges de Miséricorde. Des images de villes apparaissent précocement dans les communes du Nord de l'Italie (voir les exemples cités par Marshall 2000 et Frugoni 1983: 80-81).

Dans un tableau votif peint par Benedetto Bonfigli pour la confrérie des Gonfalons en 1464, Pérouse est figurée au registre inférieur, au pied d'une Vierge de Miséricorde: on y voit la ville close et au-delà de ces murailles les pestiférés fauchés par la mort. Ce tableau votif conjugue ainsi la représentation du mal et la réponse que lui donne la communauté, par les mesures de claustration, par la prière et l'intercession. Elle conjugue aussi deux manières de représenter le corps de la cité; le portrait architectural de Pérouse et le portrait de sa population malade. Au registre supérieur, en effet, sous le manteau de la Vierge, les fidèles se massent, hommes, femmes, clercs, laïcs, en une étendue de silhouettes agenouillées se confondant. On mesure alors ce que la 
contagion fait à la ville en frappant les corps de tous les habitants des mêmes symptômes et des mêmes émotions; elle révèle la communauté en tant que véritable corps social, les corps particuliers étant agrégés par la souffrance et par l'espoir d'une délivrance divine.

Le type des portraits votifs de cité délaisse pourtant la représentation de la communauté coagulée pour se concentrer sur la description du lieu. Les images rarement conservées, la documentation évoque la commande de quelques unes figurant simplement la ville elle-même; ainsi en 1476, pendant la peste, la commune de Recanati offre une maquette de la ville en argent à Notre-Dame de Lorette (Golsenne 2002 : 154). Dans de mêmes circonstances, les capitouls de Toulouse font réaliser en 1527 une maquette en cire, dont on peut encore voir la reproduction en bois, réalisée au Xviı ${ }^{e}$ siècle, suspendue dans le déambulatoire de la basilique Saint-Sernin.

Parvenus jusqu'à nous plus sûrement, plusieurs tableaux votifs représentent un intercesseur offrant à une figure sainte l'image de la ville, ainsi une représentation d'Ascoli, dans l'Annonciation de Crivelli, offerte par le saint évêque Emidio à la Vierge, conformément à un vœu formulé par les Anciens lors d’une épidémie (lire Golsenne 2002 : 153). Ces images présentent généralement cette forme de maquettes, figurant la muraille et les principales élévations d'une cité miniaturisée, tenant dans la main de l'intercesseur et rappelant l'iconographie du don d'église.

Le portrait votif de Besançon semble adopter une autre grammaire visuelle, celle des vues cavalières de cité, s'approchant des plans, dont Cesare De Seta note le développement au cours du XvI ${ }^{\mathrm{e}}$ siècle, notamment dans des «recueils de villes» gravés qui contribuent fortement à la construction des identités urbaines (De Seta 2011). Quand la maquette ramasse la ville derrière ses rassurantes murailles, reconnaissable par sa silhouette et puissante par sa verticalité, ici Besançon s'étale, décrivant l'ampleur de son assise dans le territoire. Le tableau en cire de 1544 présentait-il le même niveau de détail que la peinture qui le remplace en 1615? On ne peut pas l'assurer, mais en établissant le plan de la ville plutôt qu'en figurant le peloton des fidèles, le tableau de 1544 s'inscrit dans la continuité ontologique des ex-voto anatomiques d'argent ou de cire. En effet, certaines images votives précisent la structure d'un organe malade, même quand il s'agit d'un organe interne; ainsi un poumon de cire conservé au Mucem détaille soigneusement le dessin des bronches et des veines afin d'assurer l'efficacité de la représentation votive par la puissance d'une ressemblance naturaliste. Sur le même mode, le tableau de 1544 s'attache à décrire avec minutie tout ce que Besançon a dans le ventre, le lotissement du territoire urbain, l'entrelacement de ses avenues et de ses ruelles. Il s'agit bien de faire le portrait du corps de la ville, mais en lui attribuant une corporéité topographique plutôt que sociale.

Ce choix iconographique répond à une nouvelle manière, anatomique, de se représenter la ville, annonçant le triomphe du plan. Il répond aussi à la nature de l'infection, car si la peste est une maladie des hommes, la pestilence est une maladie du lieu: pour les gouverneurs de Besançon, il s'agit de guérir le territoire corrompu afin d'en délivrer les habitants. Le tableau de 1544 traite donc prioritairement le corps malade du lieu.
4. Vierge de Miséricorde, Benedetto Bonfigli, 1464, peinture sur toile, ex-voto de San Francesco al Prato, Pérouse

5. Ex-voto figurant les poumons et la trachée Cire moulée et peinte, $23 \mathrm{~cm} \times 12 \mathrm{~cm}$, première moitié $d u$ xxe siècle, Marseille, Mucem

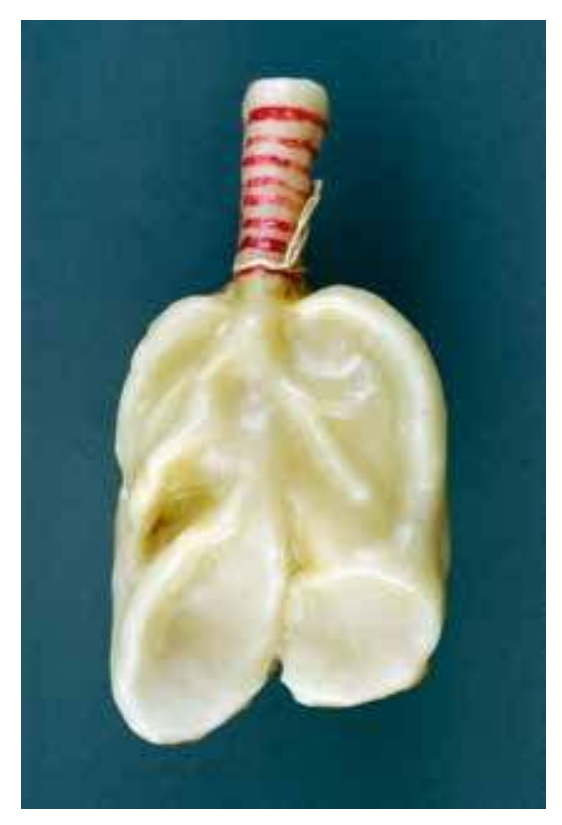




\section{Une ville dans la chapelle}

La cathédrale Saint-Étienne de Besançon a été détruite au XVII siècle, mais la documentation permet de reconstituer le mobilier de la chapelle du Saint-Suaire. Un tabernacle commandé en 1530 et placé sous un crucifix accueille le saint linge en 1534 et la relique reçoit des images et des offrandes: on a mentions d'une chaîne en or, de draps, de chandelles offerts au Saint Suaire, tandis que dès 1531 un «tronc à offrandes » est installé dont les bénéfices sont réservés à la réparation et à la décoration de la chapelle 5 . De plus, si aucun document n'éclaire la manière dont l'image a été déposée dans la chapelle, les comptes de la Ville précisent son mode de présentation; le 16 août 1544, le serrurier Jean Renotte et le maître Mattey, maçon de la Ville, reçoivent paiement après des travaux faits à Saint-Étienne «pour soustenir et interposer la cité offerte par messires audit Saint Suaire» (CC102: f॰125v) ${ }^{6}$.

\section{Reproduction d'une maquette votive de la ville de Toulouse offerte en 1527 \\ Bois, xvIII' siècle, basi- lique Saint-Sernin}

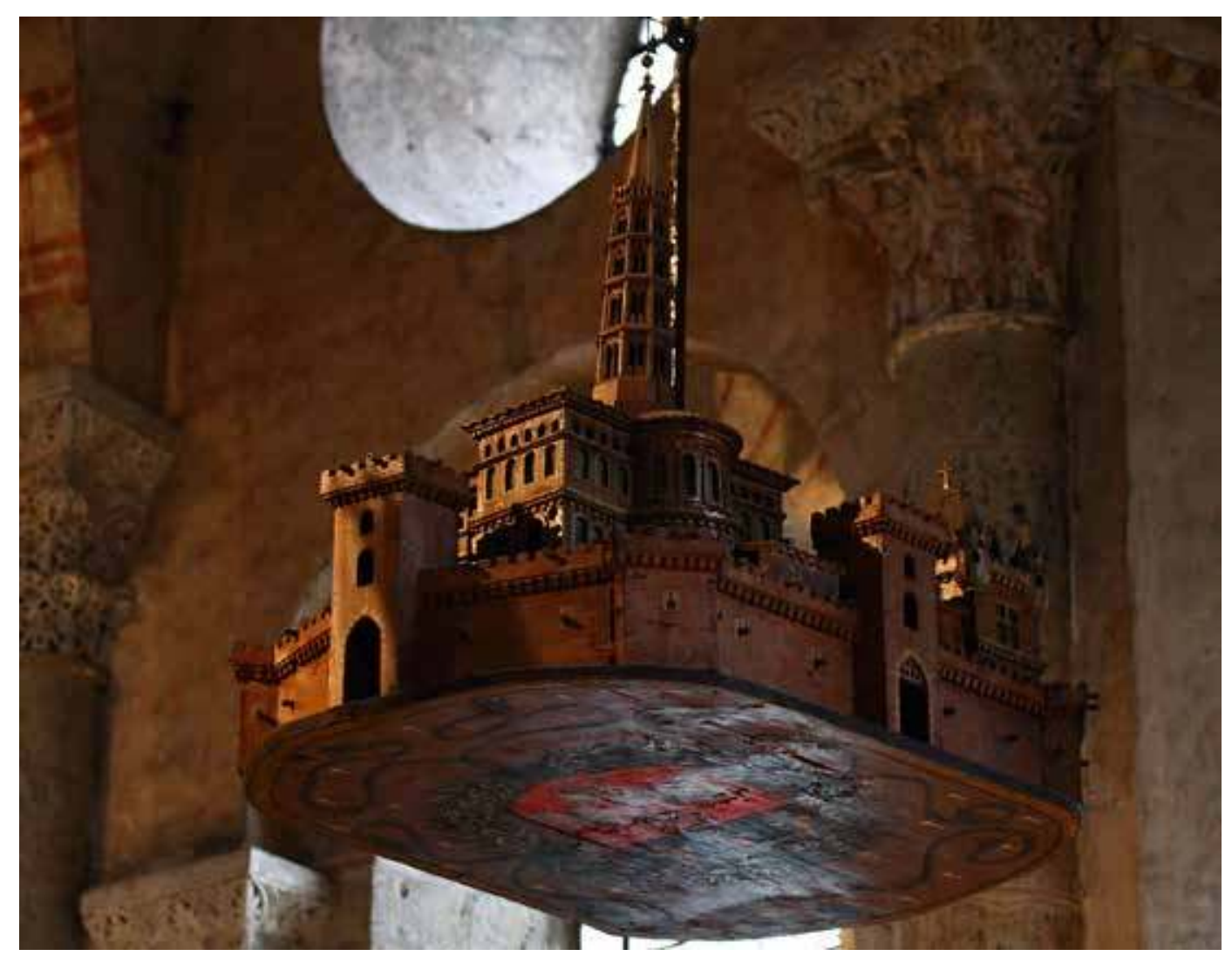




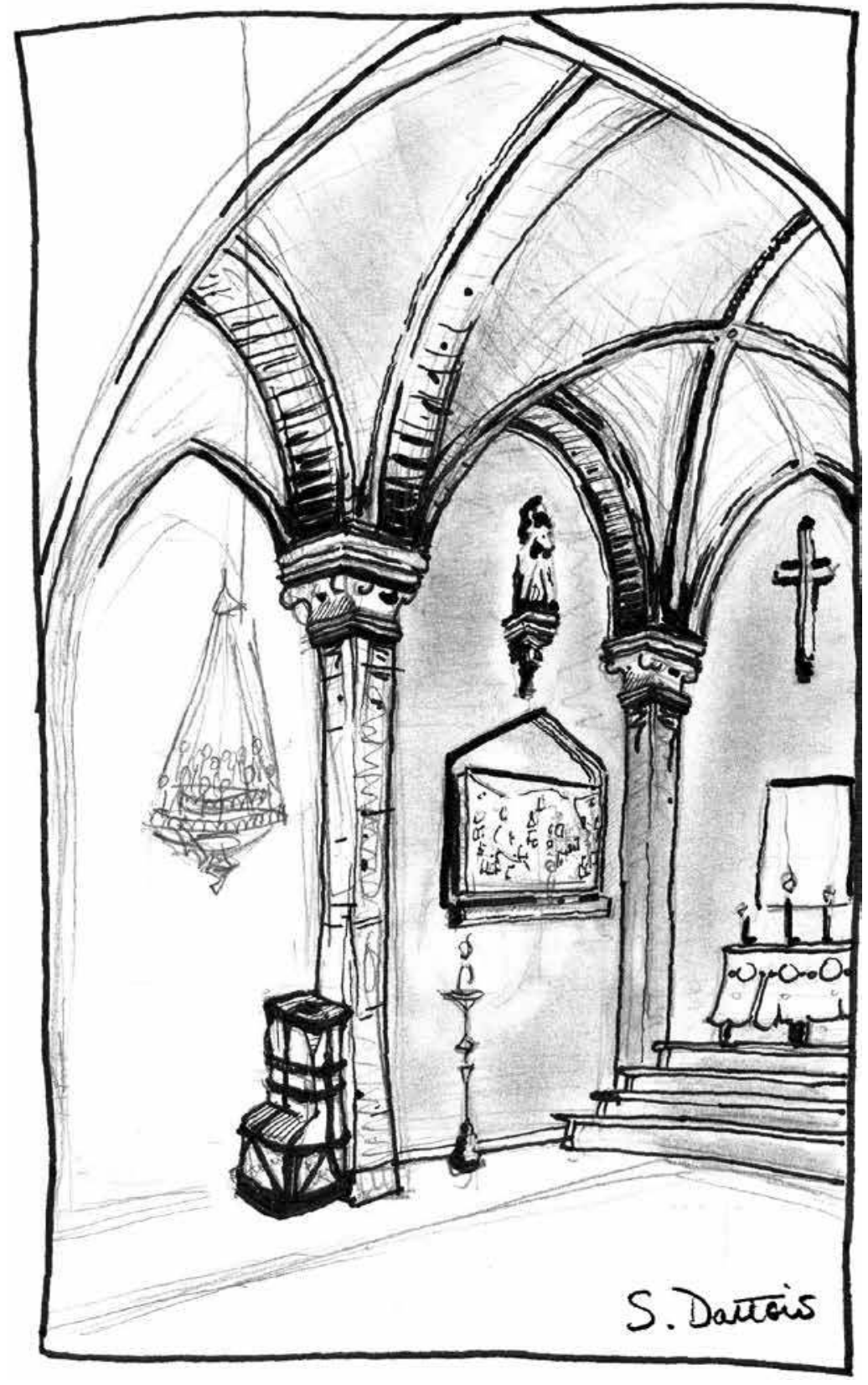

7. La relique, dans son tabernacle, au-dessus de l'autel, et la cité votive Proposition de reconstitution de la chapelle du Saint-Suaire en 1544 


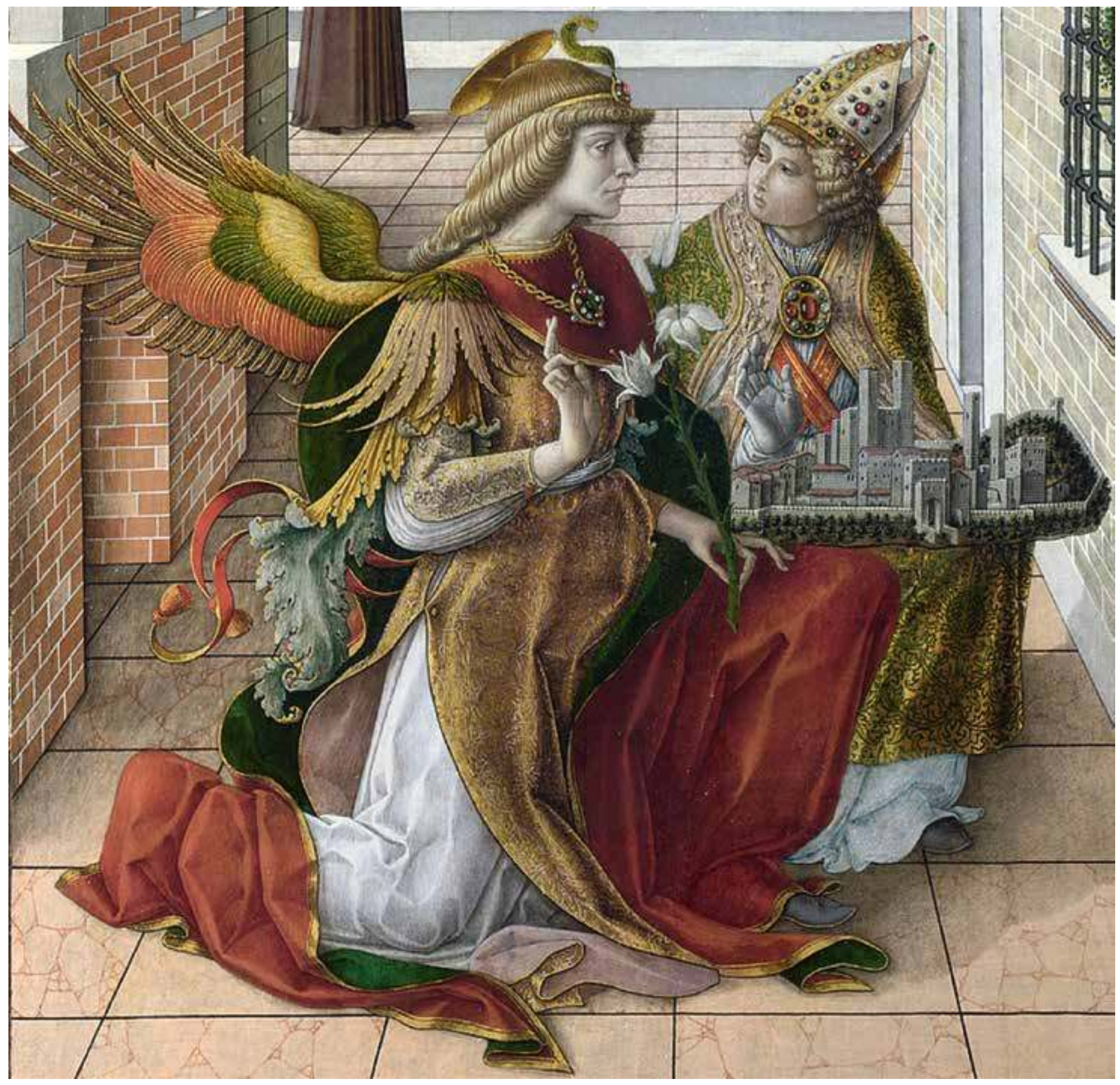


L'édification d'un dispositif surélevant la cité a plusieurs effets. D’une part le plan horizontal des offrandes du commun est rompu, donnant à l'offrande de la communauté une dignité supérieure. Ensuite, de la cité en cire au Saint Suaire, dans son tabernacle, peut s'établir un dialogue d'image, un «pacte de visibilité » (Dittmar 2015: 97) entre la communauté et la relique. L'espace de la chapelle devient dès lors un plan d'images agissantes, dans lequel se contracte et s'accomplit l'échange votif: la ville s'est vouée au Christ, l'image de la ville se donne donc, dans la chapelle, à l'image du Christ. Au corps de la ville répond un corps salutaire, d'une autre nature; l'image-relique du Christ, formée par le contact et par l'imprégnation de son sang et de ses humeurs.

Le soubassement expose aussi le tableau aux fidèles bisontins venus au Saint Suaire. Bien sûr, il s'agit de conjurer leur peur, de leur montrer une cité «triomphante», en ordre. Si on en croit le tableau de Samson Bruley, la cité est encadrée par le Doubs, les murailles, mais encore par les principales institutions; en haut se tiennent les armes de l'Empire et de la Ville, les saints de la cité, Ferréol et Ferjeux, Jean et Étienne, sont relégués dans les angles inférieurs. Dressée pour elle-même devant la relique, l'image de la cité se trouve dotée d'une personnalité agissante, elle devient un intercesseur séculier. Dans la vue de Besançon, offerte en lieu et place d'une Vierge de Miséricorde, les murailles et les armes de la cité se donnent comme manteau protecteur, au-delà duquel la mort fauche. Elle dit aux visiteurs: il vaut mieux être dans l'image qu'en dehors.

Car, et c'est le dernier effet de l'exposition de ce tableau saturé de représentations, la cité offerte, à l'instar de l'iconographie du don d'église dont elle reprend les codes, produit un transport du fidèle dans l'espace de l'image. En regardant la réduction de sa ville et le détail de l'église dans laquelle il se tient, le fidèle est aspiré par le tableau votif, incorporé à la représentation et donné lui aussi, quelque part dans l'image, au Saint Suaire. Par cette analogie, les gouverneurs s'exposent comme représentants et protecteurs de la cité, elle tient dans leurs mains. Initiateurs et donateurs de l'image, ils s'exposent, en dernier ressort, comme fondateurs ${ }^{7}$.

\section{Après la crise}

Dans la ville impériale de Besançon, le jeu est serré entre le chapitre et la municipalité. En 1534, l'archevêque écrit à Charles Quint: «Les dits gouverneurs monstrent toujours plus ouvertement le désir qu'ils ont de ruiner et effacer la juridiction ecclésiastique. » (Ms.9: fo226v.) Au prisme des querelles territoriales, l'exposition par la Ville du plan de Besançon dans l'espace de la cathédrale apparaît comme un coup politique remarquable. Le chapitre, s'il a approuvé la fondation en juin 1544 (G194 : fo362-365), a bien conscience de l'ouverture consentie aux gouverneurs, 
9. Saint-Étienne de

Besançon

Détail de la Vue de Besançon d'après Pierre d'Argent le Vleux, gravure publiée en 1575 qui peuvent désormais faire célébrer des offices réguliers dans la chapelle du Saint-Suaire. Au cours de difficiles tractations pour rédiger les articles réglant la confrérie, actés le 22 avril 1547 (voir la charte de fondation, BB407), les chanoines s'efforcent de prémunir l'Église d'un possible «préjudice pour sa liberté et son immunité » (mai 1545, G194 : fo492v). En avril 1546, ils demandent à «être instituteurs de ladite confrérie seuls, sans y mettre lesdits sires gouverneurs, sinon en qualité de confrères ». Les gouverneurs refusent, rappelant que le vœu a été réalisé «en nom de toute la cite» $\left(\mathrm{BB} 24: \mathrm{f}^{\circ} 271 \mathrm{v}\right)^{8}$.

Si le chapitre a accepté le principe d'une confrérie qu'il lui faut désormais contenir, c'est parce que la peste a cessé en 1545. La crise constitue en effet un temps politique singulier, les mesures d'exception prises pour endiguer l'épidémie formant ce que Leonardo Fioaravanti nomme en 1571 un régime de peste; sous la contagion, Églises et États jouent l'unité (Cohn 2009: 283-293). Mais les avantages gagnés dans l'urgence demeurent durablement. Après 1544, non seulement les gouverneurs font célébrer annuellement des offices au Saint Suaire, mais ils s'investissent de plus en plus, politiquement et financièrement, dans l'organisation des ostensions de la relique; la Ville a percé dans la sphère du chapitre.

En août 1576, la peste frappe de nouveau. Les gouverneurs font «redresser le tableau de la cité en cire qu'avoit esté voué à l'institution de la confrairie du sainct suayre» (BB36: f०58r). Ils le font «pourter en la chapelle»; s'il n'était pas resté auprès de la relique, il avait visiblement été conservé. Jean-Jacques Chifflet, érudit proche des gouverneurs, narre en 1624 dans son Discours des suaires la cessation subite de la peste grâce au vœu de 1544. Il écrit:

« Il y a un tableau de ce miracle dedans l'Église de sainct Estienne, avec une inscription au nom du Senat et du peuple de Besançon.» (1631 [1624] : 70.)

Il s'agit du tableau de Samson Bruley, commandé par les gouverneurs et installé à leur demande dans la chapelle du Saint-Suaire en mai 1615. Les notes de Chifflet mentionnent en effet la substitution, en 1615, de l'ancien tableau votif par un nouveau, avec l'inscription: «SPQB EX VOTO» (ms. 51 : f०16v et 54v, voir aussi CC107: f॰225 et G201).

Avec cette peinture, le vœu de 1544

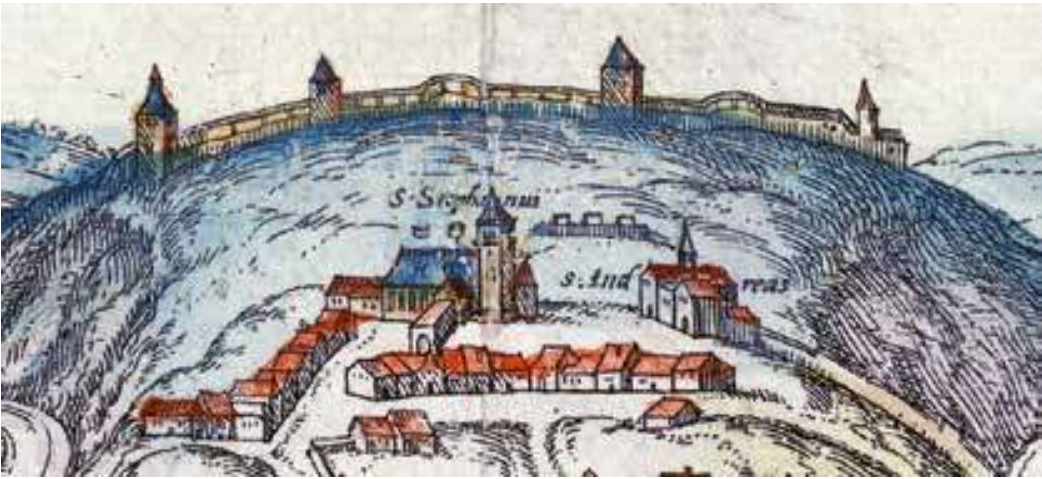
devient un épisode légendaire de l'histoire de la ville, mis au service d'une mythologie de la République bisontine. Comme dans d'autres lieux frappés par la peste au $\mathrm{XVI}^{\mathrm{e}}$ siècle, le souvenir de la «libération de pestilence» (Cohn 2009: 140) vient faire écran à celui de la crise; la calamité est transmuée en miracle, accompli par les gouverneurs et le Saint Suaire, qui gagnent leurs galons de figures tutélaires de la cité. 
Cette peinture constitue une image singulière; elle est à la fois commémoration du prodige accompli par le Saint Suaire, preuve pour la postérité de son pouvoir et de celui des gouverneurs qui l'ont sollicitée, mais l'inscription la désigne aussi comme une image votive, toujours active pour prémunir la cité de toutes formes de périls. Enfin, si ces périls devaient se préciser, l'image peut périodiquement être rechargée par un nouveau vœu. Ainsi, en février 1629, pendant la peste, les gouverneurs «confirm[ent] les anciens vœux en icelle occasion» (BB56: f232). À nouveau, en 1674, la Ville promet, si l'enceinte est libérée du siège de Louis XIV, de faire mettre dans la chapelle «le plan et portraict de la cité» (BB96: fo54v). Les gouverneurs n’auront pas à exécuter leur promesse: la ville libre d'Empire tombe aux mains du roi de France.
10. Répartition par année des dépenses de la Ville pour le culte du Saint Suaire, converties en gros (1523-1570)

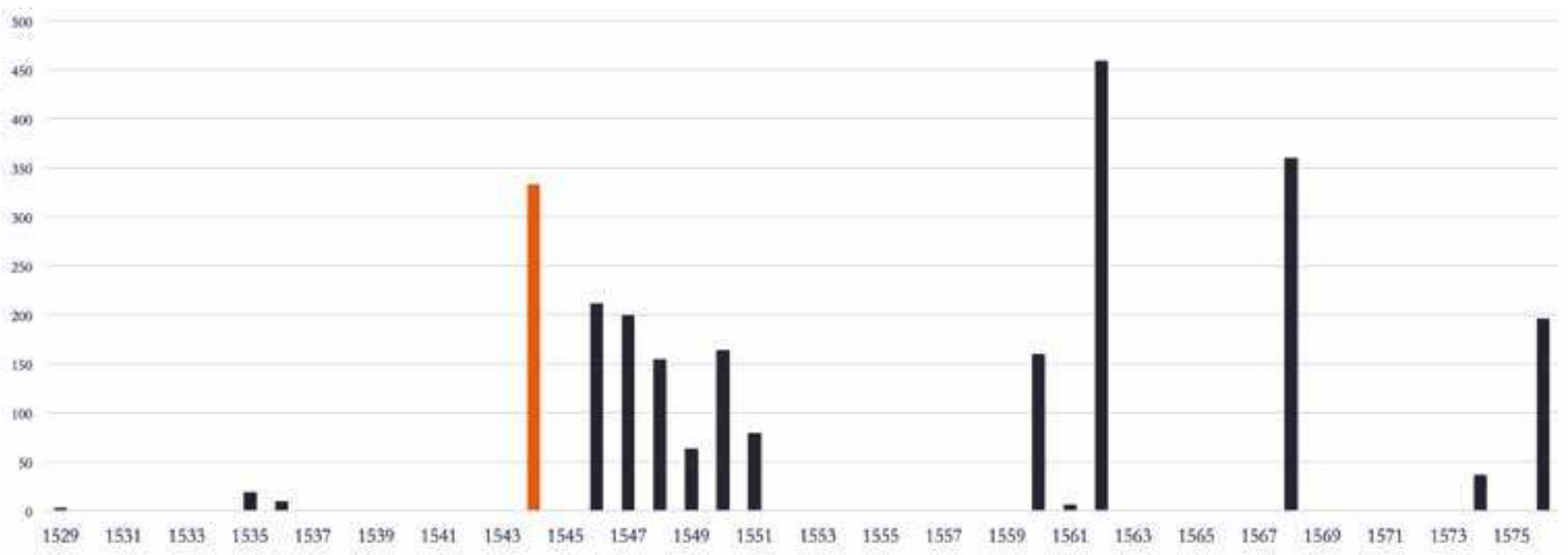

\title{
Ia peste, maladie politique
}

\author{
Albert Camus, La peste (2008 [1947] : 75-76)
}

«Une fois les portes fermées, ils s'aperçurent qu'ils étaient tous, et le narrateur lui-même, pris dans le même sac et qu'il fallait s'en arranger. C'est ainsi, par exemple, qu'un sentiment aussi individuel que celui de la séparation d'avec un être aimé devint soudain, dès les premières semaines, celui de tout un peuple, et, avec la peur, la souffrance principale de ce long temps d'exil. [...] On peut dire que cette invasion brutale de la maladie eut pour premier effet d'obliger nos concitoyens à agir comme s'ils n'avaient pas de sentiments individuels.» 
La peste est un moment propice pour brosser le portrait d'une cité: la refermant sur ellemême, elle la coagule en une communauté de peurs, de souffrances et de destins. En son sein, personne ne guérira seul. Par une double assimilation, de l'individu à la communauté et de la communauté à son territoire, l'infection fait apparaître la ville à elle-même comme un corps solidaire.

Gilles Deleuze et Felix Guattari proposent une pensée de la contagion, contre une pensée de la filiation (1972: 295-296):

«Les bandes humaines et animales prolifèrent avec les contagions, les épidémies, les champs de bataille et les catastrophes. [...] Le vampire ne filiationne pas, il contagionne. La différence est que la contagion, l'épidémie met en jeu des termes tout à fait hétérogènes: par exemple un homme, un animal et une bactérie, un virus, une molécule, un micro-organisme.»

Quand la contagion de peste tient la ville, des entités de trois natures distinctes s'hybrident pour former une totalité cohérente: le corps de l'individu, le corps social et le corps du territoire. Il fallait faire une image de cette totalité pour obtenir une guérison générale, mais après l'épreuve, il fallait pour les gouverneurs en perpétuer le souvenir; l'image-corps de la ville, produite au plus fort de la crise et vouée au Saint Suaire, en était devenue la définition idéale et indépassable.

\section{Notes}

1. Je remercie Thierry Euvrard, Marylise Forster, la bibliothèque municipale de Besançon, le musée du Temps et le Mucem pour leur accueil et pour la permission gracieuse d'utiliser leurs images. Merci à Léa Brandy, Sylvain Dartois, Sophie Degioanni et Maëlle Diarra pour leur concours, ainsi qu'à Pierre-Olivier Dittmar, Pierre Antoine Fabre, Luc Ferrier et Thomas Golsenne pour leurs précieuses orientations et remarques. Ce dossier a été présenté et s'est enrichi lors de la journée d'étude du 9 mars 2018 et lors des séances du séminaire ex-voto, à l'EHESS, j'en remercie les organisateurs et les participants. J'adresse des remerciements particuliers à Dominique et André Couton. Cette enquête est dédiée à Elvira.

2. BB24, fo73v: «Messires les gouverneurs susnommez accompaignez de plusieurs des vingtieme [les notables élus des principaux quartiers de Besançon] sont allez a sainct Estienne oyr messe devant le precieux sainct Suayre et eut offrir, au nom de tout

le corps de la cite, une cité en cyre, afin qu'il pleut a Dieu appaiser son ire et faire cesser la peste en nostre cite. Dieu doint [comprendre «daigne» ?] que ainsi soit, amen.»

3. Mentionné comme peintre par les chanoines en février 1532 (G193, f²30), Girard d'Argent reçoit de la Ville, en mars 1544, 4 francs pour «quatre evangilles escriptes en parchemins». Il reçoit encore 5 sous d'or en février 1549 pour «avoir pourtraict la cité pour icelluy envoyer a Basle pour imprimer » (CC102, f106, CC109, f80). Il devient finalement contrôleur des monnaies en 1559; sur la famille d'Argent, voir Brault-Lerch 1976 : 156-162. Ce portrait de la ville imprimé à Bâle est sans doute une reprise de l'iconographie de la cité en cire de 1544 et un prototype de la vue de Besançon telle qu'on la retrouve gravée par Hans-Rudolf Manuel, et publiée en 1575 (image d'ouverture).

4. En 1576, les gouverneurs redressent «le tableau de la cité en cire quant este voue a l'institution de 
la confrarie du sainct suayre», BB36, f०60r, tandis qu'autour de 1615 les notes de l'érudit Jean-Jacques Chifflet évoquent les évènements de 1544 et le «tableau votif (tabula votiva)» qui fut alors installé dans la chapelle, ms. 51, f०54r.

5. Pour le tabernacle, au-dessus de l'autel (Chifflet 1631 [1624] : 52) et «subpedibus crucifixi » voir G193: f $171 v, 184 v$ et 373 . Les oblations sont mentionnées au fo79r, 83r (1528), 230v, 264r (1532), le tronc à offrandes, dévolues à la réparation de la chapelle, est désigné «truncum » lors de son installation en 1531 puis « tronci sive capsule » lors d'une réparation en 1541 (ibidem: fo220v-221r, fo586r).

6. Les deux artisans se partagent 3 gros et 3 blancs, soit environ un quart de franc, pour 31 livres de fer (un peu plus de 15 kilos si on se conforme à une livre parisienne de 489 grammes) mises en œuvres afin de soutenir la cité. Le compte précisant le paiement d'une seule journée de travail et le faible coût de l'intervention caractérisent une installation sommaire. Il peut s'agir d'un soubassement léger pour «soustenir et interposer (poser entre, intercaler) la cité» ou d'un simple mode de suspension consolidé par la structure en fer. On peut imaginer que la cité votive se trouvait sur un mur latéral de la chapelle, en face du Saint Suaire, ou encore sur le mur de l'au- tel, au plus près de la relique dans son tabernacle. À noter qu'en 1576, pour «avoir dressé le modelle de la cité», le maître maçon Richard Maire reçoit la somme beaucoup plus conséquente de 16 francs, 4 gros, 3 blancs (CC130, fo 126r). Cette seconde installation était sans doute plus fastueuse et pérenne que la première.

7. Je me nourris ici des réflexions de Pierre Antoine Fabre sur le «don d'église» pour la journée d'études du Care du 16 juin 2013, «Images fondatrices», dont on attend la publication.

8. « de modo erigendo confratria sancti sudarii sine preiudicio ecclesie et libertatum ac immunitatem eiusdem», écrivent les chanoines le 8 mai 1545 (G194: fo492v). Ils entendent être nommés instituteurs de la confrérie mais veulent aussi s'assurer du bénéfice des offrandes faites au Saint Suaire dans le cadre de la confrérie. Dans les actes de 1547, le chapitre obtient gain de cause, les chanoines étant finalement nommés instituteurs de la confrérie au nom des gouverneurs et de la ville (voir G195, fo30 à 35 et les actes de la confrérie, datés du 22 avril, conservés aux archives municipales de Besançon, cote GG407). En pratique toutefois, les gouverneurs apparaissent bien comme les véritables initiateurs et organisateurs des offices de la confrérie.

\section{I'auteur}

Nicolas Sarzeaud est doctorant en histoire de l'art à l'École des hautes études en sciences sociales, sous la direction d'Étienne Anheim et de Pierre-Olivier Dittmar. Il étudie la reproductibilité des images, s'intéressant notamment aux saints Suaires et aux saintes Faces, à leurs modalités d'ostension et à leurs reproductions à la fin du Moyen Âge. Il étudie par ailleurs les pratiques de substitution d'œuvres d'art par des fac-similés, notamment les grottes préhistoriques de Lascaux et Chauvet et les moulages de sculptures des jardins de Versailles.

\section{Iconographie}

Image d'ouverture. Vue de Besançon. Gravure de Hans Rudolf Manuel d'après Pierre d'Argent le Vieux, chalcographie coloriée, publiée en 1575. C Archives municipales de Besançon.

1. BB24, f116. () Archives municipales de Besançon.

2. Musée alsacien. Photographie: Ji-Elle, Wikimedia Commons.

3. Besançon, musée du Temps. (C) Photographie: Yves Sancey.

4. Photographie Georges Jansoon, Wikimedia Commons.
5. (C) Mucem/Christophe Fouin.

6. Photographie Pierre Selim, Wikimedia Commons.

7. (C) Dessin de Sylvain Dartois.

8. (C) The National Gallery, London / National Gallery Picture Library.

9. Vue de Besançon, 1575 (détail) (C) Archives municipales de Besançon.

10. (C) Nicolas Sarzeaud. 


\section{Documents}

Archives municipales de Besançon.

BB15-96 «Délibérations des gouverneurs 1533-1674».

BB407 «Actes de fondation de la confrérie du Saint Suaire de Besançon, 22 avril 1547 ».

CC83-166 «Compte des gouverneurs 1523-1615».

\section{Bibliographie}

Baschet, J. 1994 «Image et événement : l'art sans la Peste (1348-1400)? », La Peste nera : dati di una realtà ed elementi di una interpretazione. Todi: Convegno del Centro di Studi sul Basso Medioevo di Todi: 25-47.

Brault-Lerch, S. 1976 Les Orfèvres de Franche-Comté. Genève: Droz.

Camus, A. 2008 [1947] La Peste. Paris: Gallimard.

Chifflet, J.-J. 1631 [1624] Hiérothonie ou discours des suaires de Notre Seigneur. Paris: Cramoisy.

Cohn, S. 2009 Cultures of Plague. Medical Thinking at The End of the Renaissance. Oxford: Oxford University Press.

Deleuze, G. \& F. Guattari 1972 Mille plateaux. Capitalisme et schizophrénie. Paris: Minuit.

Delsalle, P. 2004 La Franche-Comté au temps de Charles Quint. Besançon: Presses universitaires de Franche-Comté.

De Seta, C. 2011 Ritratti di città. Dal Rinascimento al secolo XVIII. Turin: Einaudi.

Didi-Huberman, G. 2006 Ex-voto. Images, organe, temps. Paris: Bayard.

\section{Pour citer l'article}

Sarzeaud, N. 2018 «La ville, corps malade: Besançon 1544», TechniquesE Culture 70 « Matérialiser les désirs. Techniques votives», p. 80-97.
GG407 «Actes de la confrérie du précieux Saint Suaire $1547 »$.

Ms. Chifflet 9, 50, 51, « Documents sur l'histoire de Besançon et du Saint Suaire».

Archives départementales du Doubs.

G192 à 201, « Délibérations capitulaires 1519-1618».

Dittmar, P.-O. 2015 «Cachez ce saint que je ne saurais voir. Modifications de visibilité en contexte rituel au Moyen Âge», Cahiers d'anthropologie sociale 11 : 84-99.

Frugoni, C. 1983 Una lontana città. Sentimenti e immagini nél Medioevo. Turin: Einaudi.

Golsenne, T. 2002 «L'Annonciation de Carlo Crivelli et le problème de l'ornement », Studiolo 1: 149-176.

Hesse, H. 2010 [1930] Narcisse et Goldmund. Paris: Librairie générale française.

Marshall, L. 2000 «Confraternity and Community. Mobilizing the Sacred in Times of Plague » in B. Wisch $\&$ D. Cole dir. Confraternities and the Visual Arts in the Press: 20-45.

Meiss, M. 2013 [1951] La peinture à Florence et à Sienne après la Peste noire. Paris: Hazan.

Nicolotti, A. 2015 Le Saint Suaire de Besançon et le chevalier Othon de La Roche. Besançon: Franche-Bourgogne. Italian Renaissance. Cambridge: Cambridge University 
